\title{
On the performance of different synchronization measures in real data: a case study on EEG signals
}

\author{
R. Quian Quiroga ${ }^{\dagger *}$, A. Kraskov ${ }^{\dagger}$, T. Kreuz ${ }^{\dagger \dagger}$, and P. Grassberger ${ }^{\dagger}$ \\ $\dagger$ John von Neumann Institute for Computing, \\ Forschungszentrum Jülich GmbH, \\ D - 52425 Jülich, Germany \\ ${ }^{\ddagger}$ Department of Epileptology, University of Bonn, \\ Sigmund-Freud Str. 25, \\ D - 53105 Bonn, Germany
}

May 28, 2018

PACS numbers: 05.45.Tp; 87.90.+y; 87.19.Nn 05.45.Xt

* corresponding author 


\begin{abstract}
We study the synchronization between left and right hemisphere rat EEG channels by using various synchronization measures, namely non-linear interdependences, phase-synchronizations, mutual information, cross-correlation and the coherence function. In passing we show a close relation between two recently proposed phase synchronization measures and we extend the definition of one of them. In three typical examples we observe that except mutual information, all these measures give a useful quantification that is hard to be guessed beforehand from the raw data. Despite their differences, results are qualitatively the same. Therefore, we claim that the applied measures are valuable for the study of synchronization in real data. Moreover, in the particular case of EEG signals their use as complementary variables could be of clinical relevance.
\end{abstract}




\section{Introduction}

The concept of synchronization goes back to the observation of interactions between two pendulum clocks by Huygens. Synchronization of oscillatory systems has been widely studied but it was not until recently that synchronization between chaotic motions received attention. A first push in this direction was the observation of identical synchronization of chaotic systems [1, 2, 3, [4. But more interesting has been the idea of a "generalized synchronization" relationship as a mapping between non-identical systems, and the further proposal by Rulkov et al. [5] of a topological method to quantify it. The work of Rulkov and coworkers indeed triggered a number of studies applying the concept of generalized synchronization to real data. One of these applications is to the study of electroencephalographic (EEG) signals, where synchronization phenomena have been increasingly recognized as a key feature for establishing the communication between different regions of the brain [6], and pathological synchronization as a main mechanism responsible of an epileptic seizure [7]. Since many features of EEG signals cannot be generated by linear models, it is generally argued that non-linear measures are likely to give more information than the one obtained with conventional linear approaches.

In a first study dealing with EEG signals, Schiff and coworkers [8] applied a synchronization measure similar to the one defined in [5] to the study of data from motoneurons within a spinal cord pool. More recently, nonlinear synchronization measures were used for the analysis of EEG data from epileptic patients with the main goal of localizing the epileptogenic zone and of predicting the seizure onset [9, 10, [1]. These results, of course, have a clear clinical relevance. Arnhold and coworkers [11] proposed a robust measure $(S)$, 
a variant of which $(H)$, already mentioned by these authors, has been studied in detail in [12]. These last two measures of interdependence, together with a new measure $(N)$ to be defined, will be further studied in this paper.

The previous papers give convincing arguments in favor of using nonlinear interdependences, which in most cases were illustrated with examples using chaotic toy models. However, it still remains an open question whether this also holds true for real data. In this paper we therefore address the point of whether non-linear measures give a relevant contribution to the study of synchronization in electroencephalographic (EEG) signals 13. In particular, we will show with three typical EEG examples (see Fig. 1) how non-linear interdependence measures can disclose information difficult to obtain by visual inspection. Although the data are EEG recordings from rats, their main features are common to human EEG. Moreover, results should not be restricted to EEG data and should also be valuable to study synchronization of other signals. For comparison purposes, we will also study phase synchronization measures as defined from the Hilbert transform [14] and from the Wavelet transform [15], which had been recently applied to the study of EEG signals [16, 18, 19]. Moreover, we will also compare these results with the ones obtained with more conventional measures of synchronization, such as the cross-correlation, the coherence function and the mutual information.

The paper is organized as follows: In section 2 we define the synchronization measures to be used. In particular, in section 2.1 we define the linear cross-correlation and the coherence function, while in section 2.2 we describe three recently proposed measures of non-linear interdependence. The mutual information is defined in section 2.3, whereas section 2.4 is dedicated to the description of phase synchronization measures with the phases defined from a Hilbert transform. Very close to these last measures are the ones described 
in section 2.5 but in this case the phases are defined from the Wavelet transform. Finally in section 2.6 we show the relation between these two phase synchronization approaches. Details of the data sets to be analyzed are disclosed in section 3. In section 1 we describe the results obtained by applying the different synchronization measures to these data sets. Finally in section 5 we present the conclusions.

\section{Synchronization measures}

In the following, unless when further specified, we shall use the notion of synchronization in a very loose sense. Thus it is more or less synonymous with interdependence or (strong) correlation.

\subsection{Linear measures of synchronization}

Let us suppose we have two simultaneously measured discrete univariate time series $x_{n}$ and $y_{n}, n=1, \ldots, N$. The most commonly used measure of their synchronization is the cross-correlation function defined as:

$$
c_{x y}(\tau)=\frac{1}{N-\tau} \sum_{i=1}^{N-\tau}\left(\frac{x_{i}-\bar{x}}{\sigma_{x}}\right) \cdot\left(\frac{y_{i+\tau}-\bar{y}}{\sigma_{y}}\right)
$$

where $\bar{x}$ and $\sigma_{x}$ denote mean and variance, and $\tau$ is a time lag. The crosscorrelation gives a measure of the linear synchronization between $x$ and $y$. Its absolute value ranges from zero (no synchronization) to one (maximum synchronization) and it is symmetric: $c_{x y}(\tau)=c_{y x}(\tau)$.

The sample cross-spectrum is defined as the Fourier transform of the cross-correlation or, via the Wiener-Khinchin theorem, as:

$$
C_{x y}(\omega)=(\mathcal{F} x)(\omega) \cdot(\mathcal{F} y)^{*}(\omega)
$$


where $(\mathcal{F} x)$ is the Fourier transform of $x, \omega$ are the discrete frequencies $(-N / 2<\omega<N / 2)$ and ${ }^{*}$ means complex conjugation. For details of the implementation, see sec. 4.1. The cross-spectrum is a complex number whose normalized amplitude

$$
\Gamma_{x y}(\omega)=\frac{\left|C_{x y}(\omega)\right|}{\sqrt{C_{x x}(\omega)} \cdot \sqrt{C_{y y}(\omega)}},
$$

is called the coherence function and gives a measure of the linear synchronization between $x$ and $y$ as a function of the frequency $\omega$. This measure is very useful when synchronization is limited to some particular frequency band, as it is usually the case in EEG signals (see [20] for a review).

\subsection{Non-linear interdependences}

From time series measured in two systems $\mathbf{X}$ and $\mathbf{Y}$, let us reconstruct delay vectors 21] $\mathbf{x}_{n}=\left(x_{n}, \ldots, x_{n-(m-1) \tau}\right)$ and $\mathbf{y}_{n}=\left(y_{n}, \ldots, y_{n-(m-1) \tau}\right)$, where $n=1, \ldots N, m$ is the embedding dimension and $\tau$ denotes the time lag. Let $r_{n, j}$ and $s_{n, j}, j=1, \ldots, k$, denote the time indices of the $k$ nearest neighbors of $\mathbf{x}_{n}$ and $\mathbf{y}_{n}$, respectively.

For each $\mathbf{x}_{n}$, the squared mean Euclidean distance to its $k$ neighbors is defined as

$$
R_{n}^{(k)}(\mathbf{X})=\frac{1}{k} \sum_{j=1}^{k}\left(\mathbf{x}_{n}-\mathbf{x}_{r_{n, j}}\right)^{2}
$$

and the $\mathbf{Y}$-conditioned squared mean Euclidean distance is defined by replacing the nearest neighbors by the equal time partners of the closest neighbors of $\mathbf{y}_{n}$ see fig. 2,

$$
R_{n}^{(k)}(\mathbf{X} \mid \mathbf{Y})=\frac{1}{k} \sum_{j=1}^{k}\left(\mathbf{x}_{n}-\mathbf{x}_{s_{n, j}}\right)^{2}
$$


If the point cloud $\left\{\mathbf{x}_{n}\right\}$ has an average squared radius $R(\mathbf{X})=\frac{1}{N} \sum_{n=1}^{N} R_{n}^{(N-1)}(\mathbf{X})$, then $R_{n}^{(k)}(\mathbf{X} \mid \mathbf{Y}) \approx R_{n}^{(k)}(\mathbf{X}) \ll R(\mathbf{X})$ if the systems are strongly correlated, while $R_{n}^{(k)}(\mathbf{X} \mid \mathbf{Y}) \approx R(\mathbf{X}) \gg R^{(k)}(\mathbf{X})$ if they are independent. Accordingly, we can define an interdependence measure $S^{(k)}(\mathbf{X} \mid \mathbf{Y})$ [1] as

$$
S^{(k)}(\mathbf{X} \mid \mathbf{Y})=\frac{1}{N} \sum_{n=1}^{N} \frac{R_{n}^{(k)}(\mathbf{X})}{R_{n}^{(k)}(\mathbf{X} \mid \mathbf{Y})}
$$

Since $R_{n}^{(k)}(\mathbf{X} \mid \mathbf{Y}) \geq R_{n}^{(k)}(\mathbf{X})$ by construction, we have

$$
0<S^{(k)}(\mathbf{X} \mid \mathbf{Y}) \leq 1
$$

Low values of $S^{(k)}(\mathbf{X} \mid \mathbf{Y})$ indicate independence between $\mathbf{X}$ and $\mathbf{Y}$, while high values indicate synchronization (reaching maximum when $S^{(k)}(\mathbf{X} \mid \mathbf{Y}) \rightarrow 1$ ).

Following ref. [11, 12] we define another non-linear interdependence measure $H^{(k)}(\mathbf{X} \mid \mathbf{Y})$ as

$$
H^{(k)}(\mathbf{X} \mid \mathbf{Y})=\frac{1}{N} \sum_{n=1}^{N} \log \frac{R_{n}(\mathbf{X})}{R_{n}^{(k)}(\mathbf{X} \mid \mathbf{Y})}
$$

This is zero if $\mathbf{X}$ and $\mathbf{Y}$ are completely independent, while it is positive if nearness in $\mathbf{Y}$ implies also nearness in $\mathbf{X}$ for equal time partners. It would be negative if close pairs in $\mathbf{Y}$ would correspond mainly to distant pairs in $\mathbf{X}$. This is very unlikely but not impossible. Therefore, $H^{(k)}(\mathbf{X} \mid \mathbf{Y})=0$ suggests that $\mathbf{X}$ and $\mathbf{Y}$ are independent, but does not prove it. This is one main difference between $H^{(k)}(\mathbf{X} \mid \mathbf{Y})$ and the mutual information, to be defined in sec. 2.3. The latter is strictly positive whenever $\mathbf{X}$ and $\mathbf{Y}$ are not completely independent. As a consequence, mutual information is quadratic in the correlation $P(\mathbf{X}, \mathbf{Y})-P(\mathbf{X}) P(\mathbf{Y})$ for weak correlations $(P$ are here probability distributions), while $H^{(k)}(\mathbf{X} \mid \mathbf{Y})$ is linear. Thus $H^{(k)}(\mathbf{X} \mid \mathbf{Y})$ is more sensitive to weak dependences which might make it useful in applications. Also, it should be easier to estimate than mutual informations which are notoriously hard to estimate reliably as we will see later. 
In a previous study with coupled chaotic systems [12], $H$ was more robust against noise and easier to interpret than $S$, but with the drawback that it is not normalized. Therefore, we propose a new measure $N(\mathbf{X} \mid \mathbf{Y})$ using also a different way of averaging,

$$
N^{(k)}(\mathbf{X} \mid \mathbf{Y})=\frac{1}{N} \sum_{n=1}^{N} \frac{R_{n}(\mathbf{X})-R_{n}^{(k)}(\mathbf{X} \mid \mathbf{Y})}{R_{n}(\mathbf{X})}
$$

which is normalized (but as in the case of $H$, it can be slightly negative) and in principle more robust than $S$.

The opposite interdependences $S(\mathbf{Y} \mid \mathbf{X}), H(\mathbf{Y} \mid \mathbf{X})$ and $N(\mathbf{Y} \mid \mathbf{X})$ are defined in complete analogy and they are in general not equal to $S(\mathbf{X} \mid \mathbf{Y})$, $H(\mathbf{X} \mid \mathbf{Y})$ and $N(\mathbf{Y} \mid \mathbf{X})$, respectively. The asymmetry of $S, H$ and $N$ is the main advantage over other non-linear measures such as the mutual information and the phase synchronizations defined in sections 2.4 and 2.5 . This asymmetry can give information about driver-response relationships [11, 12, 22], but can also reflect the different dynamical properties of each data [11, 12]. To address this point we will compare results with synchronization values obtained from time shifted signals used as surrogates.

Figure 2 illustrates the idea of how the non-linear interdependence measures work. Let us consider a Lorenz and a Roessler system that are independent (upper case, no coupling) and a second case with the Roessler driving the Lorenz via a strong coupling (lower plot). For a detailed study of synchronization between these systems refer to [12]. Given a neighborhood in one of the attractors, we see how this neighborhood maps in the other. If the point cloud is still a small neighborhood (lower plot), the systems are synchronized. On the other hand, if the points are spread over the attractor (upper plot), the systems are independent. The three measures described $S$, $H$ and $N$, are just different ways of normalizing these ratio of distances. 


\subsection{Mutual Information}

The previous measures of synchronization were based on similarities in the time and frequency domain (sec. 2.1) or on similarities in a phase space (sec. 2.2). In this section we describe an approach to measure synchronization by means of information-theoretic concepts. Let us suppose we have a discrete random variable $X$ with $M$ possible outcomes $X_{1}, \ldots, X_{M}$, obtained e.g. by a partition of $X$ into $M$ bins. Each outcome has a probability $p_{i}, i=1, \ldots, M$, with $p_{i} \geq 0 \forall i$ and $\sum p_{i}=1$. A first estimate is to consider $p_{i}=n_{i} / N$, where $n_{i}$ is the number of occurrences of $X_{i}$ after $N$ samples. From this set of probabilities the Shannon entropy is defined as:

$$
I(X)=-\sum_{i=1}^{M} p_{i} \log p_{i}
$$

The Shannon entropy is positive and measures the information content of $X$, in bits, if the logarithm is taken with base 2 . When finite samples $N$ are considered, the naive definition $p_{i}=n_{i} / N$ may not be appropriate. Grassberger [23] introduced a series of correction terms, which are asymptotic in $1 / N$. The first and most important term essentially gives

$$
I(X) \approx \sum_{i} \frac{n_{i}}{N}\left(\log N-\Psi\left(n_{i}\right)\right)
$$

with $\Psi(x)=d \log \Gamma(x) / d x \approx \log x-1 / 2 x$ for large $x$.

Let us now suppose we have a second discrete random variable $Y$, whose degree of synchronization with $X$ we want to measure. The joint entropy is defined as:

$$
I(X, Y)=-\sum_{i, j} p_{i j}^{X Y} \log p_{i j}^{X Y}
$$


where $p_{i j}^{X Y}$ is the joint probability of $X=X_{i}$ and $Y=Y_{j}$. If the systems are independent we have $p_{i j}^{X Y}=p_{i}^{X} \cdot p_{j}^{Y}$ and then, $I(X, Y)=I(X)+I(Y)$. Thus, the mutual information between $X$ and $Y$ is defined as

$$
M I(X, Y)=I(X)+I(Y)-I(X, Y)
$$

which indicates the amount of information of $X$ we obtain by knowing $Y$ and vice versa. If $X$ and $Y$ are independent, $M I(X, Y)=0$ and otherwise, it will take positive values with a maximum of $M I(X, X)=I(X)$ for identical signals. Note also that $M I$ is symmetric, i.e. $M I(X, Y)=M I(Y, X)$. Schreiber extended the concept of $M I$ and defined a transfer entropy [24], which has the main advantage of being asymmetric and can in principle distinguish driver-response relationships. Another asymmetric measure based on the $M I$ has been proposed by Palus 25$]$.

Mutual information can also be regarded as a Kullback-Leibler entropy [26, 27], which is an entropy measure of the similarity between two probability distributions. To illustrate this, we rewrite eq.(13) in the form

$$
M I(X, Y)=\sum p_{i j}^{X Y} \log \frac{p_{i j}^{X Y}}{p_{i}^{X} \cdot p_{j}^{Y}}
$$

Then, considering a probability distribution $q_{i j}^{X Y}=p_{i}^{X} \cdot p_{j}^{Y}$ (which is the correct probability distribution if the systems are independent), eq. (14) is a Kullback-Leibler entropy and measures the difference between the probability distributions $p_{i j}^{X Y}$ and $q_{i j}^{X Y}$ [28]. In other words, $M I(X, Y)$ measures how different is the true joint probability distribution $p_{i j}^{X Y}$ from another in which independence between $X$ and $Y$ is assumed.

We previously mentioned that each $p_{i}$ can be obtained by a partition of $X$. In our case, $X$ is the space of time-delay vectors $\mathbf{x}_{n}$ as in section 2.2. In 
principle, we can calculate $p_{i}$ by box counting. But it was shown in 29, 30 that the Shannon entropies (eq. (10)) can be calculated from the first order correlation integral $C^{1}(X, \delta)$, which gives more accurate results [30, 28, 31]. Thus, instead of calculating probabilities within boxes of a fixed grid with sidelength $\delta$, we compute probabilities within neighborhoods of a certain radius $\delta / 2$ around each point [30]. Therefore we have:

$$
I(X ; \delta)=-\frac{1}{N} \sum_{i=1}^{N} \log p_{i}
$$

with $p_{i} \simeq \frac{n_{i}}{N}, n_{i}=\sum_{j} \Theta\left(\delta / 2-\left\|\mathbf{x}_{i}-\mathbf{x}_{j}\right\|\right)$ and $N$ the number of embedding vectors. In this case, we can also introduce finite sample corrections which give 23

$$
I(X ; \delta)=-\frac{1}{N} \sum_{i=1}^{N}\left(\Psi\left(n_{i}+1\right)-\log N\right)
$$

\subsection{Phase synchronization from the Hilbert Transform}

Given a univariate measurement $x(t)$ (with continuous t) we first define the analytic signal $Z_{x}(t)=x(t)+i \tilde{x}(t)=A_{x}^{H}(t) e^{i \phi_{x}^{H}(t)}$, where $\tilde{x}(t)$ is the Hilbert Transform of $x(t)$ [14,

$$
\tilde{x}(t) \equiv(\mathcal{H} x)(t)=\frac{1}{\pi} P . V \cdot \int_{-\infty}^{+\infty} \frac{x\left(t^{\prime}\right)}{t-t^{\prime}} d t^{\prime},
$$

(P.V. means Cauchy principal value). Analogously, we define $A_{y}^{H}$ and $\phi_{y}^{H}$ from $y(t)$ \#. We say that the $x$ and $y$ are $n: m$ synchronized, if the $(n, m)$ phase difference of their analytic signals, $\phi_{x y}^{H}(t) \equiv n \phi_{x}^{H}(t)-m \phi_{y}^{H}(t)$, with

\footnotetext{
${ }^{1}$ In the actual implementation, where $x(t)$ and $y(t)$ are only known at discrete times, we calculate $\tilde{x}_{n}$ from the Fourier transform, as described in [14.
} 
$n, m$ some integers, remains bounded for all $t$. Thus, we define a phase synchronization index as 32, 18,

$$
\gamma_{\mathrm{H}} \equiv\left|\left\langle e^{i \phi_{x y}^{H}(t)}\right\rangle_{t}\right|=\sqrt{\left\langle\cos \phi_{x y}^{H}(t)\right\rangle_{t}^{2}+\left\langle\sin \phi_{x y}^{H}(t)\right\rangle_{t}^{2}}
$$

(brackets denote average over time). By construction, $\gamma_{H}$ will be zero if the phases are not synchronized at all and will be one when the phase difference is constant (perfect synchronization). The key feature of $\gamma_{H}$ is that it is only sensitive to phases, irrespective of the amplitude of each signal. This feature has been illustrated in [14] and following papers (see [32]) with bidirectionally coupled Rössler systems. Another important feature of $\gamma_{H}$ is that it is parameter free. However, if the signals to be analyzed have a broadband or a multimodal spectrum, then the definition of the phase can be troublesome and pre-filtering of the signals might be necessary. Of course, it should be checked that the filter to be used does not introduce phase distortions.

Tass and coworkers [16] defined another phase synchronization measure from the Shannon entropy of the distribution of $\phi_{x y}^{H}(t)$. The range of $\phi^{\prime}=$ $\phi_{x y}^{H}(\bmod , 2 \pi)$ is first divided into $M$ bins. Let $p_{k}$ be the probability that $\phi^{\prime}$ is in the bin $k$ at any random time. Then,

$$
\gamma_{\mathrm{H}-\mathrm{Sh}}=\frac{S_{\max }-S}{S_{\max }}, \quad S=-\sum_{k=1}^{M} p_{k} \cdot \ln p_{k}
$$

and $S_{\max }=\ln M$. It ranges from zero for an uniform distribution of $\phi_{x y}^{H}$, to one if the distribution is a delta function. The advantage over $\gamma_{\mathrm{H}}$ is that $\gamma_{\mathrm{H}}$ can underestimate phase synchronizations when the distribution of $\phi_{x y}^{H}$ has more than one peak. This corresponds to the case where the phase difference remains fairly stable but occasionally "jumps" between different values 17 . Although the signals are synchronized (except at the times of the jumps), 
the phases $\phi_{x y}^{H}(t)$ can cancel in the time average of eq.(18), thus giving a low $\gamma_{\mathrm{H}}$ ?. We also calculated another quantification proposed in [16] defined from conditional probabilities between $\phi_{x}^{H}(t)$ and $\phi_{y}^{H}(t)$, but results were very similar to those obtained with $\gamma_{\mathrm{H}}$ and will be not further reported.

\subsection{Phase synchronization from the Wavelet Trans- form}

Another phase synchronization measure defined from the Wavelet Transform $\left(\gamma_{\mathrm{W}}\right)$ has been recently introduced by Lachaux et al. 15, 34. It is very similar to $\gamma_{\mathrm{H}}$, with the only difference that the phases are calculated by convolving each signal with a complex wavelet function $\Psi(t)$ [33]

$$
\Psi(t)=\left(e^{i \omega_{0} t}-e^{-\omega_{0}^{2} \sigma^{2} / 2}\right) \cdot e^{-t^{2} / 2 \sigma^{2}},
$$

where $w_{0}$ is the center frequency of the wavelet and $\sigma$ determines its rate of decay (and by the uncertainty principle, its frequency span)․

The convolution of $x(t)$ and $y(t)$ with $\Psi(t)$ gives two complex time series of wavelet coefficients

$$
W_{x}(t)=(\Psi \circ x)_{(t)}=\int \Psi\left(t^{\prime}\right) x\left(t^{\prime}-t\right) d t^{\prime}=A_{x}^{W}(t) \cdot e^{i \phi_{x}^{W}(t)},
$$

\footnotetext{
${ }^{2}$ A multimodal distribution of the phases can also appear if we look e.g. for a $1: 1$ synchronization but the real relationship is $1: 2$.

${ }^{3}$ Instead of eq.(20), the authors of [15, 34 used a Morlet wavelet i.e. $\Psi(t)=e^{i \omega_{0} t}$. $e^{-t^{2} / 2 \sigma^{2}}$, which satisfies the zero mean admissibility condition of a wavelet only for large $\sigma$. Since in our case we will use a low $\sigma$ (i.e. a $\Psi$ with few significant oscillations, see sect.2.5), an additional negative term is introduced. When $\sigma$ is small, disregarding this term can introduce spurious effects, especially if the signal to be analyzed has non-zero mean or low frequency components. We do not need a normalization term in eq. (20) because we will be interested only in phases.
} 
$\left(W_{y}(t)\right.$ is defined in the same way from $\left.y(t)\right)$ from which we can again calculate the phase differences $\phi_{x y}^{W}(t) \equiv \phi_{x}^{W}(t)-\phi_{y}^{W}(t)$ and define a phase synchronization factor $\left(\gamma_{\mathrm{W}}\right)$ as in eq. (18), or from the Shannon entropy of the distribution of $\phi_{x y}^{W}(t)\left(\gamma_{\mathrm{W}-\mathrm{Sh}}\right)$ as in eq.(19)).

The main difference with the measures defined by using the Hilbert transform is that a central frequency $\omega_{0}$ and a width $\sigma$ for the wavelet function should be chosen, and therefore $\gamma_{\mathrm{W}}$ and $\gamma_{\mathrm{W} \text {-Sh }}$ will be sensitive only to phase synchronizations in a certain frequency band. In particular, DeShazer et. al. 35 recently analyzed phase synchronization in coupled laser systems defining the phases both from a Gabor (similar to eq.(20)) and a Hilbert transform. In the first case they distinguished a phase synchronization at $140 \mathrm{~Hz}$, something not seen when using the Hilbert transform. The difference between both approaches, of course, does not imply that one measure is superior to the other. There are cases in which one would like to restrict the analysis to a certain frequency band and other cases in which one would prefer to have a method that is parameter free, as $\gamma_{\mathrm{H}}$. In fact, in section 2.6 we will show that there is a close relation between both methods.

\subsection{Relation between the phase synchronization mea- sures}

In sections 2.4 we already mentioned that in some cases it might be necessary to pre-filter the signals before applying the Hilbert Transform, while for the Wavelet Transform a center frequency (and frequency width) should be chosen beforehand. In fact, the phases defined by the complex wavelet transform $\phi_{x}^{W}$ and by the Hilbert transform $\phi_{x}^{H}$ are closely related. Indeed, the real part of $W_{x}(t)$ can be considered as a band-pass filtered signal. From 
it, we can form the Hilbert transform

$$
\tilde{W}_{x}(t)=\left(\mathcal{H} \operatorname{Re}\left[\mathrm{W}_{\mathrm{x}}\right]\right)(t)
$$

and a phase by

$$
\operatorname{Re}\left[\mathrm{W}_{\mathbf{x}}\right](t)+i \tilde{W}_{x}(t)=A_{\mathrm{Re}\left[\mathrm{W}_{\mathbf{x}}\right]}^{H}(t) \cdot e^{i \phi_{\mathrm{Re}\left[\mathrm{W}_{\mathbf{x}}\right]}^{H}(t)} .
$$

Let us now recall the definition of analytic signals. A complex function $g(t)$ is an analytic signal if it satisfies $(\mathcal{F} g)(\omega)=0 \forall \omega<0$ [36]. If $g$ is analytic, then $\operatorname{Im}[g(t)]=\tilde{g}(t) \equiv(\mathcal{H} \operatorname{Re}[g])(t)$. If a wavelet function $\Psi$ is analytic, then $W_{x}(t)=(\Psi \circ x)_{(t)}$ is also analytidf. In this case $\tilde{W}_{x}(t) \equiv \operatorname{Im}\left[W_{x}(t)\right]$ and $\phi_{\mathrm{Re}\left[\mathrm{W}_{\mathrm{x}}\right]}^{H}(t) \equiv \phi_{x}^{W}(t)$, as defined in eq.(21). Since the corrected Morlet wavelet of eq. (20) is approximately analytic we have $\phi_{\mathrm{Re}\left[\mathrm{W}_{\mathrm{x}}\right]}^{H}(t) \cong \phi_{x}^{W}(t)$ to very good approximation. Since as we mentioned, $W_{x}(t)$ acts as a band pass filter of $x(t)$, then $\phi_{x}^{H}(t) \cong \phi_{x}^{W}(t)$ as long as for the first one the signal is pre-filtered with the same wavelet function used for calculating the latter.

It is important to remark that the previous result is not limited to complex Morlet wavelets and can be extended to other wavelet functions. In particular, from a real wavelet function $\Psi(t)$ we can construct an analytic signal by using the Hilbert transform, i.e. $\Psi^{\prime}(t) \equiv \Psi(t)+i(\mathcal{H} \Psi)(t)$, which satisfies that $W_{x}(t)=\left(\Psi^{\prime} \circ x\right)(t)$ is analytic. Then, from $W_{x}(t)$ we can define a phase and e.g. study the phase synchronization with another signal $y(t)$. The important advantage is that we have the freedom of defining the phase from a particular wavelet function, chosen from a dictionary of available wavelets according to the signal to be studied. This can be interesting

\footnotetext{
${ }^{4}$ Taking the Fourier Transform we get $\left(\mathcal{F} W_{x}\right)(\omega)=\left(\mathcal{F}(\Psi \circ x)_{(t)}\right)(\omega)=(\mathcal{F} \Psi)(\omega)$.
} $(\mathcal{F} x)(\omega)=0 \quad \forall \omega<0$, where we used the Fourier convolution theorem and that $\Psi$ is analytic.

${ }^{5}$ The Morlet wavelet tends to the analytic signal for large $\omega_{0}$ and low $\sigma$ [36]. 
in cases in which defining a phase from the Hilbert transform is troublesome or if conventional filters are not well suited.

\section{Details of the data}

We will analyze the synchronization between two EEG channels in three different data sets [13. The EEG signals were obtained from electrodes placed on the left and right frontal cortex of male adult WAG/Rij rats (a genetic model for human absence epilepsy) [37]. Both signals were referenced to an electrode placed at the cerebellum, they were filtered between 1-100 $\mathrm{Hz}$ and digitized at $200 \mathrm{~Hz}$.

In a previous study [39, the main objective of this set up was to study changes in synchronization after unilateral lesions with ibothenic acid in the rostral pole of the reticular thalamic nucleus. To achieve this, synchronization was first assessed visually by looking for the simultaneous appearance of spike discharges f and then it was further quantified by calculating both a linear cross-correlation and the non-linear interdependence measure $H$ defined in the previous section. For the quantitative analysis, for each rat and condition, 10 data segments pre- and 10 segments post-lesion were analyzed, five of these segments corresponding to normal EEGs and the other five containing spike discharges. The length of each data segment was 5 seconds (i.e. 1000 data points), this being the largest length in which the signals containing spikes could be visually judged as stationary. In all 7 rats studied, it was found that synchronization significantly decreased after the lesions in the reticular thalamic nucleus [39]. Moreover, changes shown with the non-

\footnotetext{
${ }^{6}$ More properly, "spike-wave discharges" but for simplicity we will call them spikes in the remaining of the paper.
} 
linear synchronization $H$ were more pronounced than those found with the cross-correlation. In the following section we will analyze in detail three of these EEG segments.

\section{Synchronization in the EEG data}

In Fig.1 we show the right and left channels of three of the (pre-lesion) EEG signals described in the previous section. The first case (example A) corresponds to a normal EEG, and in the remaining two cases the signals have spike discharges (examples B and C). Spikes usually appear due to a local synchronization of neurons in the neighborhood of the electrode at which they are recorded. Since epilepsy is related to an abnormal synchronization in the brain, spikes are usually considered as a landmark of epileptic activity. A localized appearance of spikes can delimit a zone with abnormal discharges (but this will not necessarily be the epileptic focus). On the contrary, if spikes are observed over the whole set of electrodes, abnormal synchronization is said to be global. This concept seems to be obvious, but it has some subtleties as we will see in the following. Let us analyze examples B and C. In both cases we see spikes at the left and right electrodes. As we said, this will point towards a global synchronization behavior. However, a more detailed analysis shows that the spikes of example B are well synchronized and in example C they are not. Indeed, in example $\mathrm{C}$ the spikes have slightly different time lags between the right and left channels. This is of course not easily seen in a first sight. For making clear this point, we picked up the spikes of examples B and $\mathrm{C}$ and we noted the times of their maximum for the right and left channels. We then calculated the lag between the spikes in the two channels and its standard deviation with time. For the case B, the lag was very small and 
stable, mainly between -5 to $5 \mathrm{~ms}$ (i.e. of the order of the sampling rate) and the standard deviation was of $4.7 \mathrm{~ms}$. For the case C, the lag was much more unstable and covered a larger range (between -20 to $50 \mathrm{~ms}$ ). In this last case the standard deviation was of $14.9 \mathrm{~ms}$. This shows that in example B the simultaneous appearance of spikes is correlated with a global synchronization, while in example $\mathrm{C}$ bilateral spikes are not synchronized (i.e. we have local synchronization for both channels, but no global synchronization). In the case of example A, due to its random-like appearance it is difficult to estimate the level of synchronization by visual inspection. However, we can already observe some patterns appearing simultaneously in both the left and right channels, thus showing some degree of interdependence.

Summarizing, we may say that example B seems the most "ordered" and synchronized. Among the other two examples, A looks definitely more disordered than $\mathrm{C}$, but a closer look raises doubts and a formal analysis is asked for.

\subsection{Linear measures}

The second column of Table 1 shows the zero lag cross-correlation values for the three examples. As stated in eq.(1), the calculation of the crosscorrelation requires a normalization of the data. We note that the tendency is in agreement with what we expect from the arguments of the previous section (i.e. $B>A>C$ ). However, the difference between cases $\mathrm{A}$ and $\mathrm{B}$ is relatively small. To get more insight, in Fig. 3 we plot the cross-correlation as a function of time shifts between the two channels. For the shifted versions, we used periodic boundary conditions. For large enough shifts, the synchronization will in principle be lost and the values obtained will give an estimation of the zero synchronization level, which we will call background level, and its 
fluctuation (i.e. we use the shifted versions as surrogates). We observe that the synchronization drops to a background level for shifts larger than 50 data points (i.e. $250 \mathrm{~ms}$ ). The average of this background level is zero, but the fluctuations are quite large. Taking these fluctuations as an estimation of the error, we see that cross-correlation does not distinguish between cases A and B.

We also note that the cross-correlation shows oscillations when shifting, most clearly in case B. These oscillations have the same period of the spikes and might put into doubt the idea of considering the shifted signals as surrogates. We therefore re-calculated the cross-correlation but taking the left channel signals from other data segments of the same rat (for each rat we had 5 segments with spikes and 5 of normal EEG before the lesions in the thalamus) and corresponding to the same condition (pre-lesion, normal EEG for example A and EEG with spikes for examples B and C). In all cases, the background level and its fluctuations were of the order of those shown in fig.3. This indicates that shifted signals can be used as surrogates in spite of the oscillations.

Figure 4 shows the spectral estimates for the three examples. The two upper plots correspond to the power spectra of the right and left channels and the lower plot to the corresponding coherence function (3). Each spectrum $\left(C_{x x}, C_{y y}\right.$ and $\left.C_{x y}\right)$ was estimated using the Welch technique'], i.e. the data is divided into $M$ segments and then $C_{x x}=\sum_{i=1}^{M} C_{x x, i}$. We used half overlapped segments of 128 data points tapered with a Hamming window. Example A has both in the right and left channels a power spectrum resembling a power law distribution, with its main activity concentrated between 1 -

\footnotetext{
${ }^{7}$ without this segmentation technique, the coherence function (eq.(3)) would be always equal to one.
} 
$10 \mathrm{~Hz}$. The coherence function shows a significant interaction for this range of frequencies. Examples $\mathrm{B}$ and $\mathrm{C}$ show a more localized distribution in the power spectrum. In both examples and for both channels there is a peak between $7-10 \mathrm{~Hz}$ and a harmonic at about $15 \mathrm{~Hz}$. In agreement with previously reported results [38], these peaks correspond to the spikes observed in Fig.1. We can already see from the power spectra that the matching between right and left channels in example B is much clearer than in example C. This is correlated with the larger coherence values of example B, showing a significant synchronization for almost the whole frequency range. On the other hand, the coherence is much lower for example $\mathrm{C}$ and it seems to be significant only for low frequencies (up to $6 \mathrm{~Hz}$ ). As in the case of the crosscorrelation, the coherence function for $\omega \leq 11 \mathrm{~Hz}$ does not distinguish well between examples A and B. There is only a difference for frequencies larger than $11 \mathrm{~Hz}$, but this just reflects the lack of activity in this frequency range for example A, whereas in example B it corresponds to the synchronization between the high frequency harmonics of the spikes. In the third column of Table 1 we summarize the results obtained with the coherence function. The values shown correspond to a frequency of $9 \mathrm{~Hz}$, the main frequency of the spikes in examples B and C.

\subsection{Non-linear interdependences}

For calculating the non-linear interdependence measures $S, H$ and $N$ between left and right electrodes we first reconstruct the state spaces of each signal using a time lag $\tau=2$ and an embedding dimension $m=10$. We chose this time lag in order to focus on frequencies lower than $50 \mathrm{~Hz}$ (i.e. half the Nyquist frequency) and the choice of the embedding dimension was in order to have the length of the embedding vectors about the length of the spikes. 
We further chose $k=10$ nearest neighbors and a Theiler correction for temporal correlations 40] of $T=50$. These parameters were chosen heuristically in order to maximize the sensitivity to the underlying synchronizations, but results were robust against changes of them. Table [ summarizes the results for the three examples. We will first discuss results with the non-linear measures $H$ and $N$. For both measures, example B has the highest synchronization due to the presence of phase-locked spike discharges and example $\mathrm{C}$ has a much smaller value. The synchronization of example $\mathrm{A}$ is between these values. Again, it is interesting to remark that the non-linear interdependence measures show the random looking signal of example A to be more synchronized than the one with spikes of example $\mathrm{C}$ but less than the one in $\mathrm{B}$, something surprising at a first sight, and not clearly following from the cross-correlation or the coherence as shown in section 4.1 .

As done for the cross-correlation, in Fig. 马 $^{\text {we }}$ we also plot the two non-linear synchronizations $H(R \mid L), N(R \mid L)$ and $H(L \mid R), N(L \mid R)$ as a function of time shifts between the two channels. Again, the synchronization drops to a background level for shifts larger than 50 data points (i.e. 250ms) and the background level is about zero. But in the case of $H$ and $N$ we observe that the fluctuations are much smaller than those for the cross-correlation. In fact, with $H$ and $N$ the synchronization levels of the three cases are clearly separated, while the cross-correlation does not distinguish between cases A and B. However, even though we expect example B to be the most ordered and synchronized of all (see sec. T), we do not have objective means for claiming that the difference between examples A and B is significant. So, the fact that non-linear measures are able to separate the three examples might imply a higher sensitivity of these measures in comparison with the linear measures, but it does not prove it. We also observe some asymmetries in 
$H$ and $N$, most pronounced in case $\mathrm{C}$. This might suggest that one of the signals drives the other (i.e. the focus is on one side). However, in all cases this is of the order of the asymmetries seen with the shifted signals, thus not significant.

The case for the synchronization measure $S$ is quite different. As seen in Fig. 5, for examples $\mathrm{B}$ and $\mathrm{C}$ there is a clear asymmetry between right and left channels. In contrary to $H$ and $N$, this asymmetry remains even for large time shifts between the two channels. Moreover, the background level for the three examples is between $0.1-0.2$ and not zero as with $H$. Thus, the asymmetries observed in examples B and $\mathrm{C}$ reflect more the individual properties of each channel rather than a synchronization phenomenong. Nevertheless, $H$ and $N$ were clearly more robust in this respect.

Again, in order to check for the validity of the shifted signals as surrogates, we re-calculated $H, N$ and $S$ but taking the left channel signals from other data segments. As in the case of the cross-correlation, the background level and its fluctuations were of the order of those shown in fig.

\subsection{Hilbert phase synchronization}

Prior to the estimation of the phase synchronization measures, each set of data was de-meaned. No further filtering was applied. Figure [6 shows the time evolution of the phases (upper plot) and their distribution (middle plots) for the three examples. From the time evolution of the phases we can already see that the phase of example B is clearly more stable than the other two examples (except in the last half second, as we will detail later). Examples

\footnotetext{
${ }^{8}$ As pointed out in [11], precisely such an asymmetry is expected if otherwise equal systems are coupled asymmetrically. Thus, if we expect both subsystems a priori to have the same complexity, the asymmetry of $S$ is a hint to an asymmetric coupling.
} 
$\mathrm{A}$ and $\mathrm{C}$ are not so easily differentiated, but in the middle plots we see that the phase distribution of $\mathrm{A}$ is more localized than the one of $\mathrm{C}$. The values of $\gamma_{H}$, indicated in Table 1, are in agreement with these observations and with the general tendency observed with the other synchronization measures $(B>A>C)$. The phase synchronization index defined from the Shannon entropy $\left(\gamma_{\mathrm{H}-\mathrm{Sh}}\right.$, defined in eq.(19) $)$ shows qualitatively similar results (see Table (1).

Since by applying the Hilbert Transform we can calculate an 'instantaneous phase' of the signals, we expect to achieve a very good time resolution with the phase synchronization measures derived from them. In the lowest plot of Fig. 6 we show the time evolution of $\gamma_{\mathrm{H}}$ (the plot for $\gamma_{\mathrm{W}-\mathrm{Sh}}$ was qualitatively similar). Each point is calculated for a window of 100 data points. In the first 3 seconds we observe relatively stable synchronization values for cases $\mathrm{A}$ and $\mathrm{B}$. For the example $\mathrm{C}$ we observe a larger variability due to a progressive phase desynchronization with a phase reentrainment at about second 2.5. For all the examples, synchronization levels oscillate around the average values noted in Table 1. After the third second the situation changes. Example $\mathrm{C}$ becomes more synchronized than the other two examples and example B gets more desynchronized in the last half a second. This is in agreement with what we see in the original signals in Fig.11, where it would have been hard to discern at a first sight by visual inspection. The possibility to follow phase synchronization in time is in fact one advantage over the non-linear interdependences, where a large number of data points is required for reasonably stable results. 


\subsection{Wavelet phase synchronization}

In this case, for calculating the phase of each signal we used a corrected Morlet wavelet (eq.(20) ) with $w_{0}$ between 1 and $30 \mathrm{~Hz}$ and $\sigma=n / 6 \omega_{0}$, where $n$ is the number of significant oscillations of the wavelet function at the $1 \%$ level. We tested different values of $n$ but in the following results with $n=1$ and $n=3$ will be shown. Larger values of $n$ led to a very bad time resolution as we detail later. We used zero padding border conditions and varied $\omega_{0}$ at $1 \mathrm{~Hz}$ intervals.

The phase difference plots (at $10 \mathrm{~Hz}$ ) were indeed very similar to those shown in Fig. 6 and will not be discussed further. Figure 7 shows the phase synchronization values $\gamma_{\mathrm{W}}$ (left plots) and $\gamma_{\mathrm{W}-\mathrm{Sh}}$ (right plots) calculated with a wavelet function containing 1 significant oscillation ( $n=1$; upper plots) and 3 significant oscillations ( $n=3$; lower plots). The values reported in Table 1 correspond to those obtained with $n=1$ at a frequency of $10 \mathrm{~Hz}$ (the frequency of the spikes in examples $\mathrm{B}$ and $\mathrm{C}$, but results are qualitatively the same between $5-15 \mathrm{~Hz}$ ). These results are very similar to those obtained with the Hilbert transform and show the same tendency (i.e. $B>A>C$ ). However, we also note that synchronization values are a bit larger than the ones of $\gamma_{H}$ and $\gamma_{\mathrm{H}-\mathrm{Sh}}$. As already shown in section 2.6, the difference is due to the frequency band selectivity of $\gamma_{\mathrm{W}}$ and $\gamma_{\mathrm{W}-\mathrm{Sh}}$. We therefore expect that a pre-filtering of the signals will increase the synchronization values calculated by using the Hilbert Transform.

With $n=1$ the three cases are well differentiated both by $\gamma_{\mathrm{W}-\mathrm{Sh}}$ and $\gamma_{\mathrm{W}}$. With $n=3$ the differences between the synchronization levels of examples $\mathrm{A}$ and $\mathrm{B}$ is less clear for $\gamma_{\mathrm{W}}$ and $\gamma_{\mathrm{W}-\mathrm{Sh}}$. This is due to the decrease in time resolution when increasing the number of significant oscillations of the mother function. Clearly, for the examples studied, $n=1$ had the best per- 
formance (for $n>3$ results get worse than for $n=3$ ). Notice the similarity between the lower plots for $n=3$, i.e. the ones with less resolution, with the coherence plots shown in Fig. 4. This supports the usefulness of the phase synchronization measures defined from the Wavelet Transform in comparison with traditional approaches. Finally, we should also remark that, as shown in section 2.6, we are not limited to use Morlet wavelets, but we can rather choose between several wavelet functions depending on the application.

\subsection{Mutual information}

Let us finally analyze the results obtained with mutual information for the three EEG signals. For its calculation we used eq. (13) with each Shannon entropy calculated by means of the correlation sum (using maximum norm) and the finite samples correction of eq. (16). After each data set was normalized, for embedding the data we used a time lag $\tau=2$ and embedding dimensions ranging from $m=1$ (no embedding) to $m=50$. We further used a Theiler correction [40] of 10 data points and for calculating the correlation sum we varied the radius $\delta$ from 0.01 to 0.5 in steps of 0.01 . In figure 8 we show the results for $m=1,2,3,4$, the results for larger $m$ had a similar tendency (see below). The difficult point when calculating $M I$ is to have a good estimation of the joint probabilities $p_{i j}^{X Y}$ (see eq.(12)). These joint probabilities involve a search of neighbors in a $2 m$-dimensional embedding space, and therefore it is difficult to find enough neighbors and get a good statistic for large $m$. We expect this restriction to be more relevant in the signals with spikes, due to their inhomogeneous distribution in state space.

In line with the previous argument, due to the small number of data points we could not get robust estimates of synchronization in the three examples analyzed. As seen in Fig. 8, the answer to the question of which signal 
is more and which is less synchronized dramatically depends on the choice of $m$ and $\delta$. We observe the same tendency as with the previous measures $(B>A>C)$ only for $m=1$ and $\delta>0.15$.

All previous analysis done in this paper show clear evidence that example B has the highest synchronization. For $m=1$ this is the case for $\delta>0.05$, for $m=2$ it occurs for $\delta>0.2$, for $m=3$ at $\delta>0.45$ and for $m=4$ it does not occur for the range of $\delta$ shown. In fact, there is a crossing between the synchronization values of examples $\mathrm{A}$ and $\mathrm{B}$, that takes place at larger $\delta$ for larger $m$. This simply reflects the impossibility of finding neighbors in the $2 m$-dimensional state space for small $\delta$ and/or large $m$. As mentioned before, we expect this effect to be less restrictive for the homogeneous distribution of example A. This explains why example A always shows the highest synchronization for small $\delta$.

\section{Conclusions}

We applied several linear and non-linear measures of synchronization to three typical EEG signals. Besides mutual information, which was not robust due to the low number of data points, all these measures gave a similar tendency in the synchronization levels. A similar analysis would have been impossible by visual inspection. Moreover, in one case with bilateral spikes, synchronization was much lower than expected at a first sight. Therefore, we claim that the quantification of synchronization between different EEG signals can complement the conventional visual analysis and can even be of clinical value. In particular, this is very important for the study of epilepsy [9, 10, 11, 18] and for the study of brain processes involving a synchronous activation of different areas or structures in the brain. 
In the last years, mainly two types of non-linear synchronization measures were proposed, namely, the ones based on phase relationships (phase synchronization) and the ones based on non-linear interdependences (generalized synchronization). It is interesting to remark that in our study with real data these measures gave similar results, despite their different definitions and their sensitivity to different characteristics of the signals. We also show a close similarity between phase synchronization measures based on the Hilbert and on the Wavelet Transform. In the particular case of the last one, we generalize its definition to different wavelet functions that will be more or less suitable according to the problem under investigation.

We validated the results obtained with the new non-linear measures by comparing them with those obtained with traditional methods. All measures ranked the synchronization levels of the three examples in the same way. However the separation between them was more pronounced with non-linear measures. Although we do not have objective means for claiming that the difference between the synchronization of the signals is significant, this might suggest a higher sensitivity of non-linear measures.

Although these results should not be automatically extended to other signals and problems, they also support the value of non-linear synchronization measures in real data analysis. 


\section{Acknowledgments}

We are very thankful to Dr. Giles van Luijtelaar and to Joyce Welting from NICI - University of Nijmegen, for the data used in this paper. We are also

indebt to Dr. Klaus Lehnertz, Florian Mormann and Giles van Luijtelaar for useful discussions and comments. One of us (A.K.) acknowledges support from the US civilian research development foundation for the independent states of the former Soviet Union, Award nr: REC-006. 


\section{References}

[1] H. Fujisaka and T. Yamada, Prog. Theor. Phys. 69, 32 (1983); 76, 582 (1986)

[2] T. Yamada and H. Fujisaka, Prog. Theor. Phys. 70, 1240 (1984); 72, $885(1985)$

[3] A. Pikovsky, Z. Physik B 55, 149 (1984).

[4] L.M. Pecora and T.L. Carroll, Phys. Rev. Lett. 64, 821 (1990).

[5] N.F. Rulkov, M.M. Sushchik, L.S. Tsimring, and H.D.I. Abarbanel. Phys. Rev. E 51, 980 (1995).

[6] C. Gray, P. Koenig, A. Engel and W. Singer, Nature 338, 335 (1989).

[7] E. Niedermeyer. Epileptic seizure disorders in Electroencephalography: Basic Principles, Clinical Applications and Related Fields, edited by E. Niedermeyer and F. H. Lopes da Silva (Baltimore, Williams and Wilkins 3rd ed., 1993), pp 1097.

[8] S.J. Schiff, P. So, T. Chang, R.E. Burke and T. Sauer. Phys. Rev. E 54, 6708 (1996).

[9] M. Le Van Quyen, C. Adam, M. Baulac, J. Martinerie, and F.J. Varela, Brain Research 792, 24 (1998).

[10] M. Le Van Quyen, J. Martinerie, C. Adam, and F.J. Varela, Physica D 127, 250 (1999).

[11] J. Arnhold, P. Grassberger, K. Lehnertz, and C.E. Elger, Physica D 134, 419 (1999). 
[12] R. Quian Quiroga, J. Arnhold and P. Grassberger. Phys. Rev. E, 61, $5142(2000)$.

[13] The three EEG signals to be studied can be downloaded from www.physio.mu-luebeck.de/user/rq/data.htm

[14] M. Rosenblum, A. Pikovsky and J. Kurths. Phys. Rev. Lett, 76, 1804 (1996).

[15] J. Lachaux, E. Rodriguez, J. Martinerie and F. Varela. Human Brain Mapping, 8, 194 (1999).

[16] P. Tass, M. Rosenblum, J. Weule, J. Kurths, A. Pikovsky, J. Volkmann, A. Schitzler and H. Freund. Phys. Rev. Lett, 81, 3291 (1998).

[17] M. Zaks, E. Park, M. Rosenblum and J. Kurths. Phys. Rev. Lett. 82, 4228, 1999.

[18] F. Mormann, K. Lehnertz, P. David and C.E. Elger. Physica D, 144, $358(2000)$.

[19] E. Rodriguez, N. George, J. Lachaux, J. Martinerie, B. Renault and F. Varela. Nature, 397, 430 (1999).

[20] F.H. Lopes da Silva, in Electroencephalography: Basic Principles, Clinical Applications and Related Fields, edited by E. Niedermeyer and F. H. Lopes da Silva (Baltimore, Williams and Wilkins 3rd ed., 1993), pp 1097.

[21] F. Takens, in D.A. Rand and L.S. Young, eds., Lecture Notes in Mathematics 898, page 366 (Springer, Berlin etc., 1981).

[22] A. Schmitz. Phys. Rev. E, 62, 7508 (2000). 
[23] P. Grassberger. Phys. Lett. A, 128, 369, 1988.

[24] T. Schreiber. Phys. Rev. Lett. 85, 461, 2000.

[25] M. Palus. Phys. Lett., submitted.

[26] R. Gray. Entropy and information theory. New York, Springer Verlag, 1990.

[27] R. Quian Quiroga, J. Arnhold, K. Lehnertz and P. Grassberger. Phys. Rev. E, 62, 8380, 2000.

[28] P. Grassberger, T. Schreiber and C. Schaffrath. Int.J. of Bifurcation and Chaos, 1, 521, 1991.

[29] P. Grassberger. Phys. Lett. A 97, 224, 1983.

[30] K. Pawelzik and H.G. Schuster. Phys. Rev. A, 35, 481, 1987.

[31] D. Pritchard and J. Theiler. Physica D 84, 476, 1995.

[32] M.G. Rosenblum, A.S. Pikovsky, C. Schäfer, P. Tass, and J. Kurths. In: Handbook of Biological Physics; Vol. 4, Neuro-informatics (F. Moss and S. Gielen eds.), Elsevier Science, pp. 279-321, 2000.

[33] A. Grossmann, R. Kronland-Martinet and J. Morlet. In: (Combes et al. eds.) Wavelets: Time-Frequency methods and phase space. Berlin, Springer (1989).

[34] J. Lachaux, E. Rodriguez, M. Le van Quyen, A. Lutz, J. Martinerie and F. Varela. Int. J. Bifurcation and Chaos, 10, 2429 (2000).

[35] D. DeShazer, R. Breban, E. Ott and R. Roy. Phys. Rev. Lett, in press. 
[36] L. Cohen. Time-frequency analysis. Prentice Hall, New Jersey (1995).

[37] G. van Luijtelaar and A. Coenen (eds.). The WAG/Rij rat model of absence epilepsy: Ten years of research. Nijmegen University Press, 1997.

[38] WHIM Drinkenburg, G. van Luijtelaar, WJ van Schaijk and A Coenen. Physiol. Behav. 54, 779, 1993.

[39] G. van Luijtelaar, J. Welting and R. Quian Quiroga. In: van Bemmel et al. (eds.) Sleep-wake research in the Netherlands, vol 11, pp:86-95. Dutch Society for Sleep-Wake Research, 2000.

[40] J. Theiler. Phys. Rev. A, 34, 2427 (1986). 


\begin{tabular}{|c|c|c|c|c|c|c|c|c|c|c|c|c|}
\hline Example & $c_{x y}$ & $\Gamma_{x y}$ & $S(R \mid L)$ & $S(L \mid R)$ & $H(R \mid L)$ & $H(L \mid R)$ & $N(R \mid L)$ & $N(L \mid R)$ & $\gamma_{H}$ & $\gamma_{H-S h}$ & $\gamma_{W}$ & $\gamma_{W-S h}$ \\
\hline A & 0.70 & 0.88 & 0.34 & 0.34 & 0.67 & 0.60 & 0.46 & 0.42 & 0.59 & 0.12 & 0.71 & 0.19 \\
\hline B & 0.79 & 0.86 & 0.35 & 0.28 & 1.11 & 1.30 & 0.63 & 0.69 & 0.71 & 0.18 & 0.80 & 0.28 \\
\hline $\mathrm{C}$ & 0.42 & 0.40 & 0.17 & 0.23 & 0.33 & 0.45 & 0.24 & 0.32 & 0.48 & 0.09 & 0.48 & 0.09 \\
\hline
\end{tabular}

Table 1: Synchronization values for the three examples of figure 1. $c_{x y}$ : cross-correlation; $\Gamma_{x y}$ : coherence (at $\left.9 \mathrm{~Hz}\right)$; $S(R \mid L), H(R \mid L), N(R \mid L)$ and $S(L \mid R), H(L \mid R), N(L \mid R)$ : non-linear interdependences of the right electrode on the left and viceversa; $\gamma_{H}$ and $\gamma_{H-S h}$ : phase synchronization indices defined from the Hilbert Transform (eq. (18) and (19), respectively); $\gamma_{W}$ and $\gamma_{W-S h}$ : phase synchronization indices defined from the Wavelet Transform. 

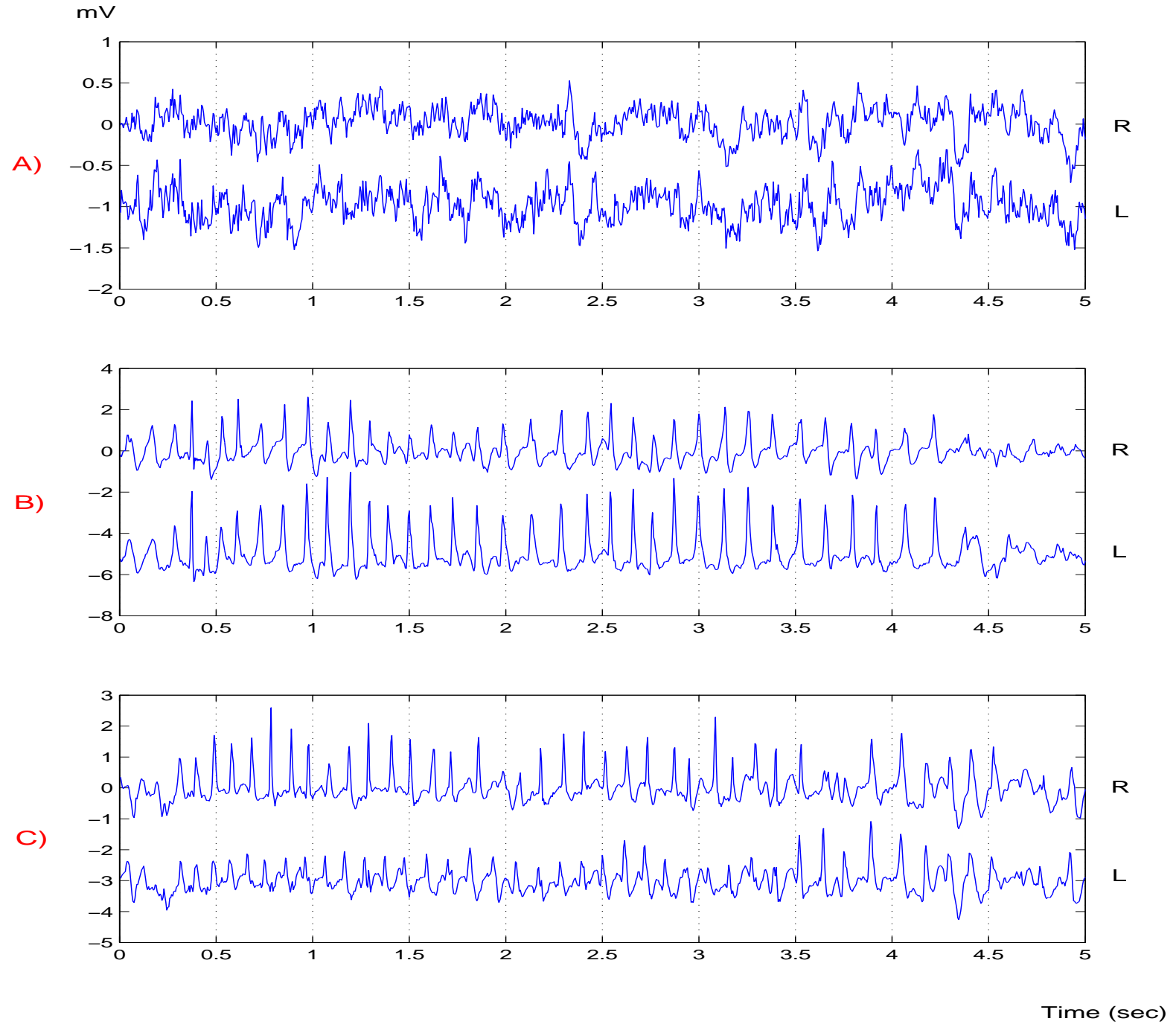

Figure 1: Three rat EEG signals from right and left cortical intracranial electrodes. For a better visualization, left signals are plotted with an offset 

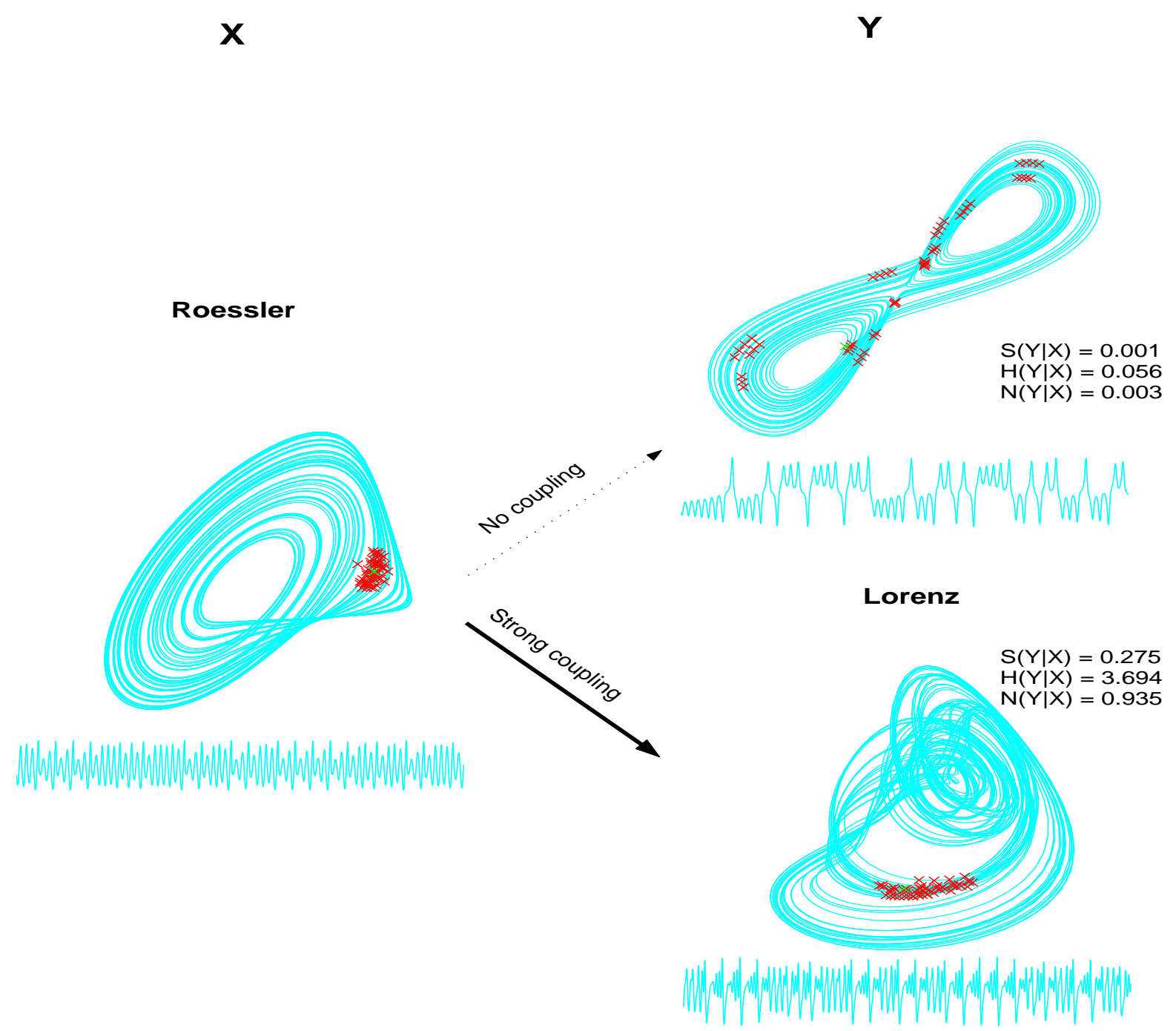

Figure 2: Basic idea of the non-linear interdependence measures. The size of the neighborhood in one of the systems, say $X$, is compared with the size of its mapping in the other system. The example shows a Lorenz system driven by a Roessler with zero coupling (upper case) and with strong coupling (lower case). Below each attractor, the corresponding time series is shown. The $(X \mid Y)$ interdependences are calculated in the same way, starting with a neighborhood in $Y$. For details see ref. [11, 12]. 

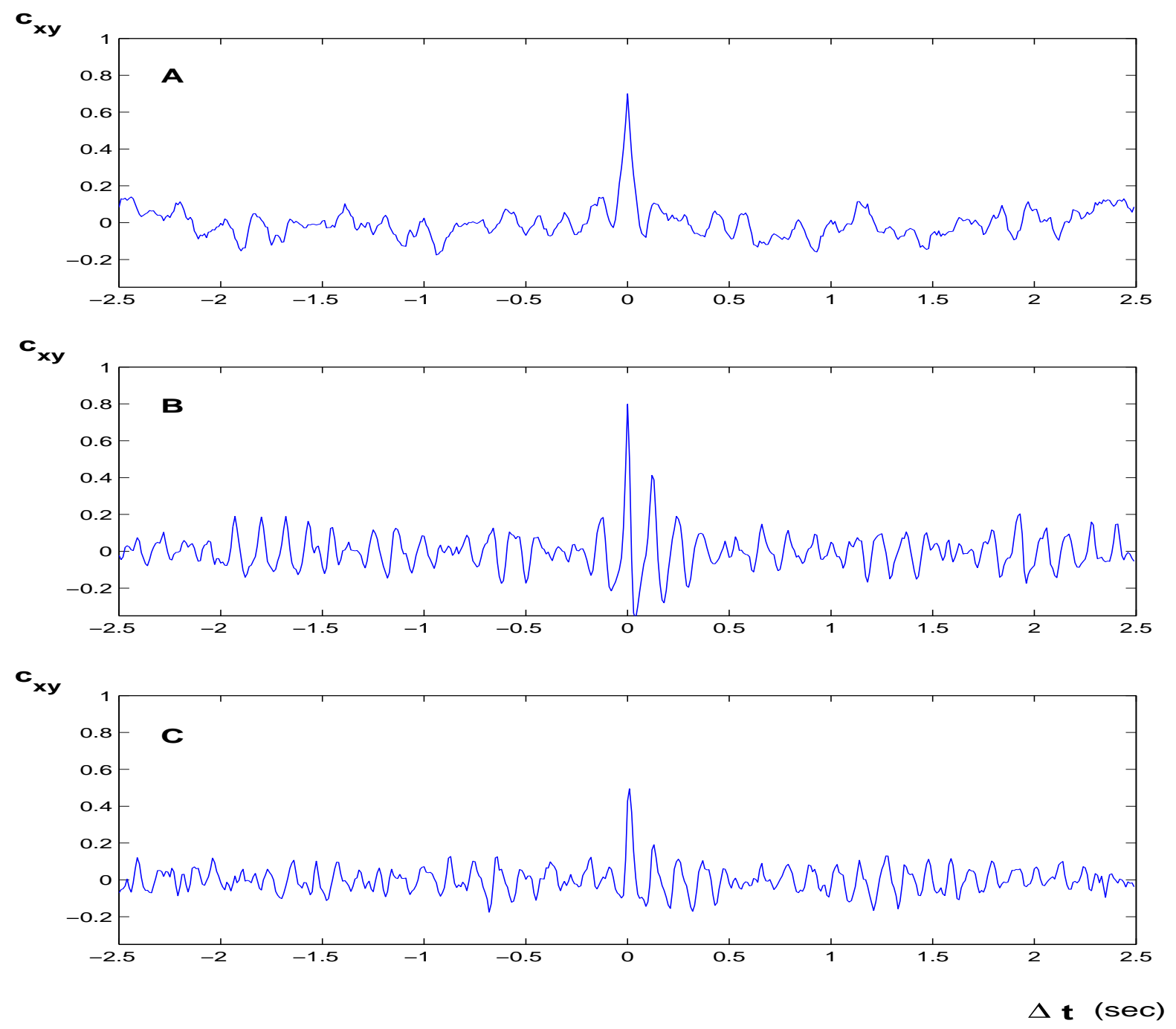

Figure 3: Cross-correlations between the right channel and shifted versions of the left one. Note that the difference between the three signals are of the order of fluctuations when shifting. 

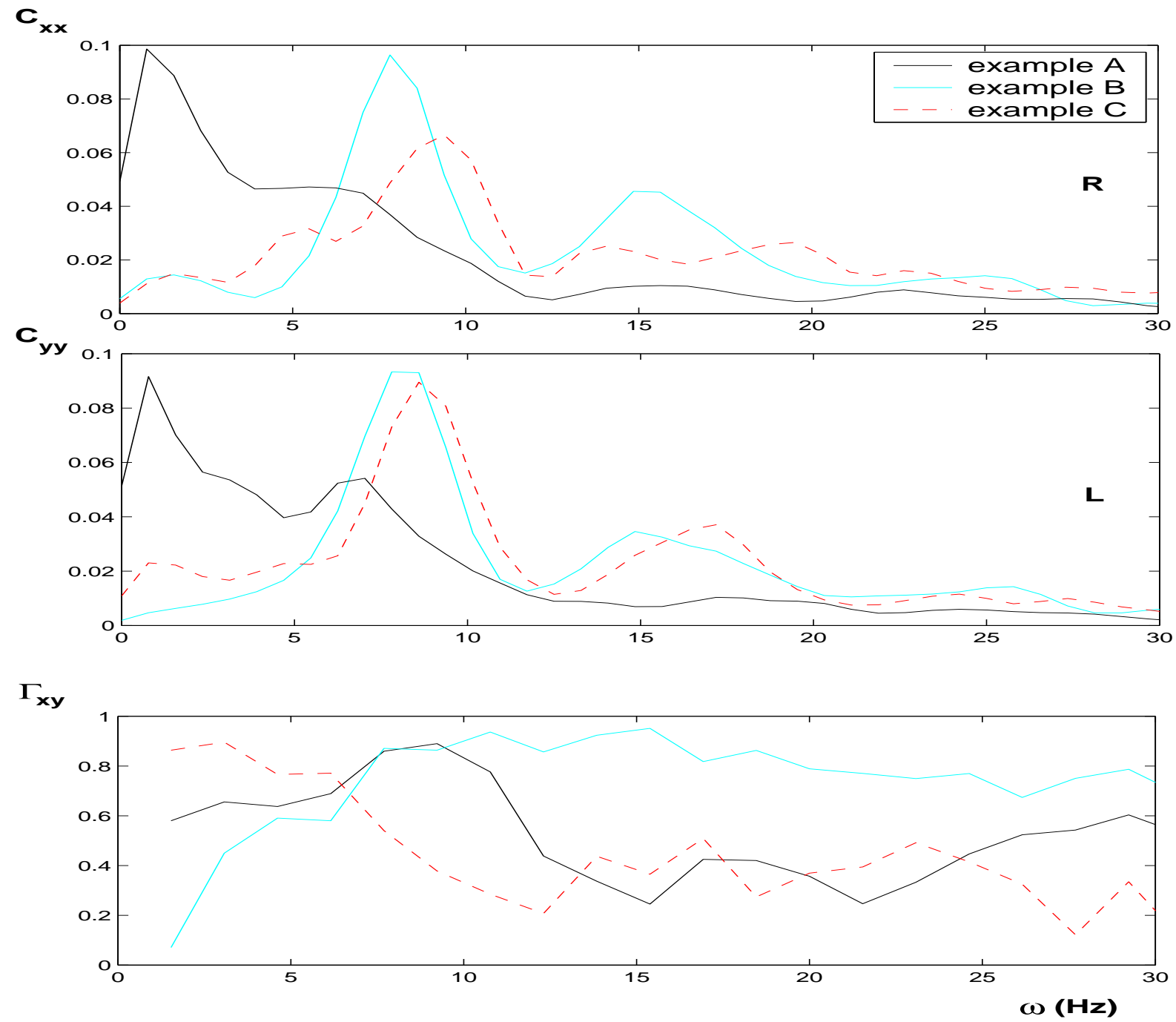

Figure 4: Power spectra of the right and left channels $\left(C_{x x}\right.$ and $C_{y y}$; upper plots) and the corresponding coherence function $\left(\Gamma_{x y}\right.$; lower plot). 

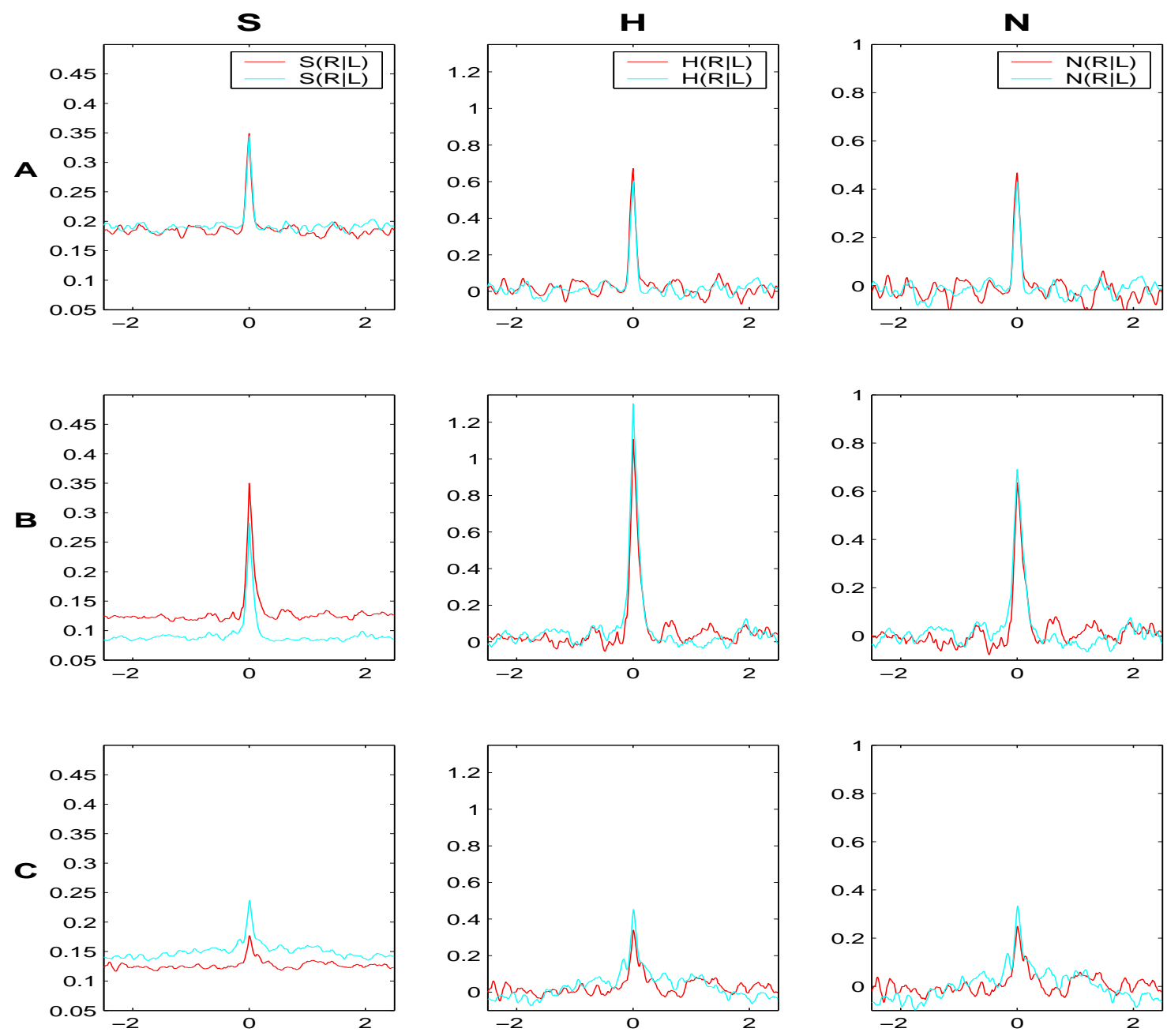

$\Delta t(\mathbf{s e c})$

Figure 5: Non-linear interdependences S, H and N between the right channel and shifted versions of the left one. Note that $\mathrm{H}$ and $\mathrm{N}$ give similar results and can distinguish the three cases. The measure $\mathrm{S}$ shows an asymmetry that remains even after shifting. See text for details. 


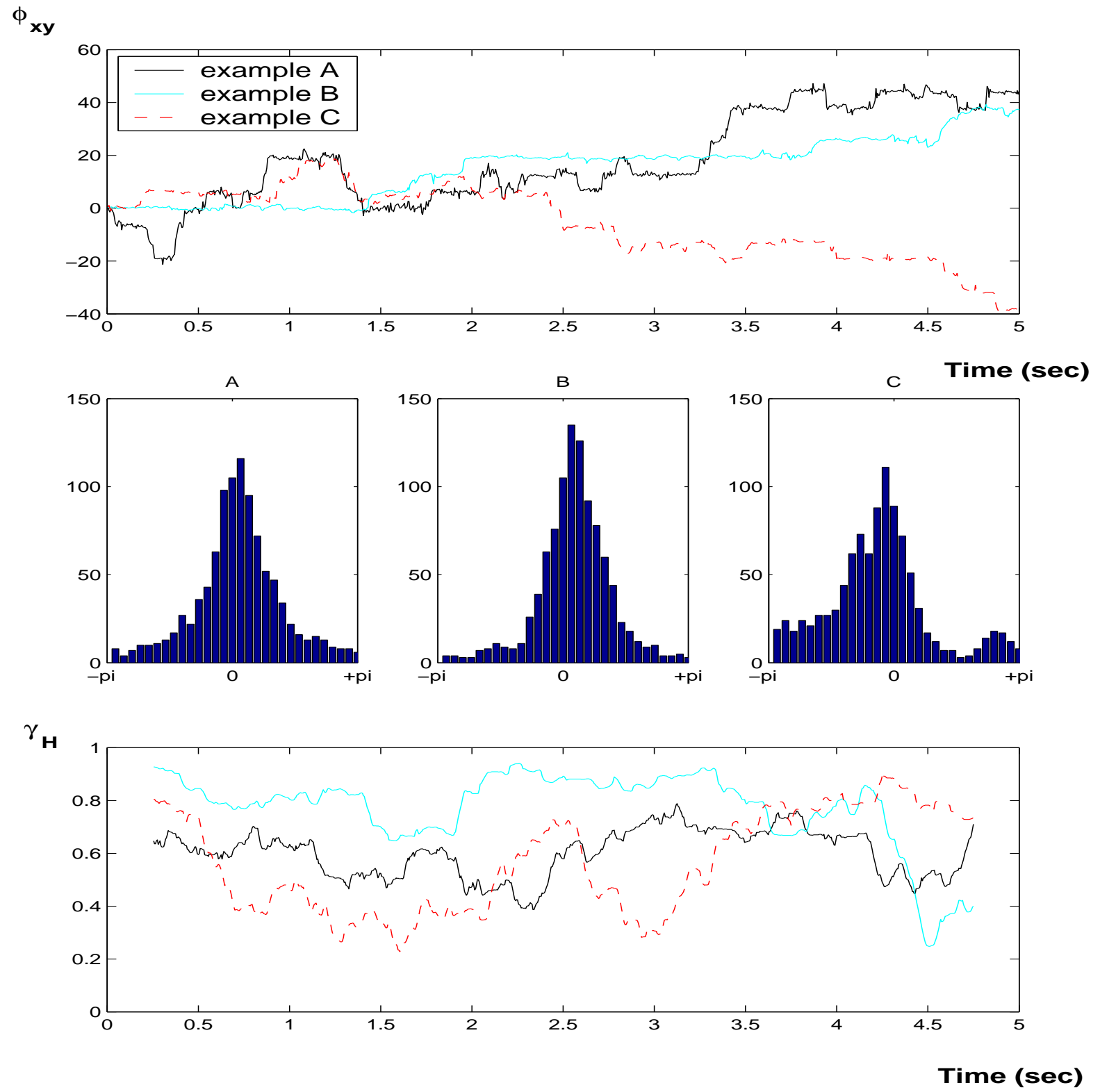

Figure 6: Time evolution of the $(1,1)$ phase differences, as defined from the Hilbert transform, for the three examples of figure 1 (upper plot), the corresponding distributions of the folded phase differences (middle plots) and the time evolution of the phase synchronization index $\gamma_{\mathrm{H}}$ (lower plot). 
$\gamma_{\mathbf{w}}$

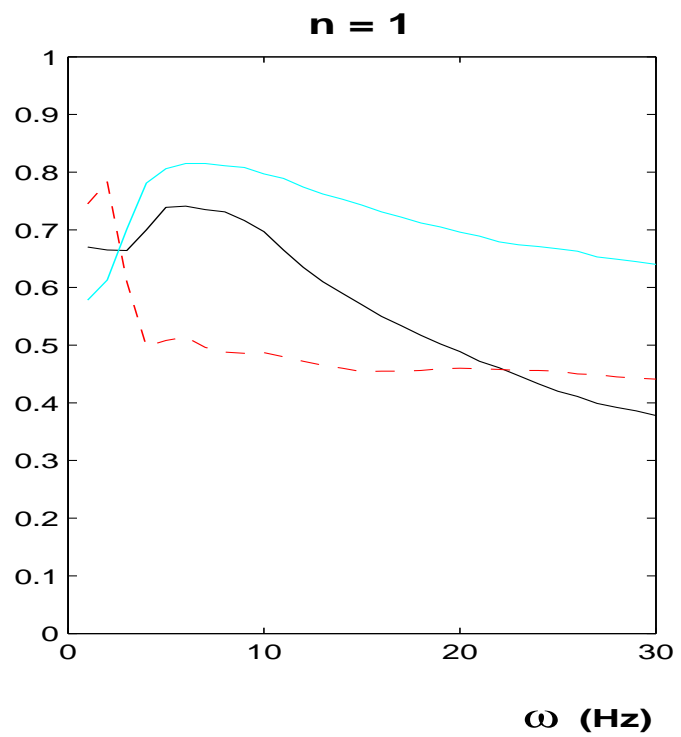

$\gamma$

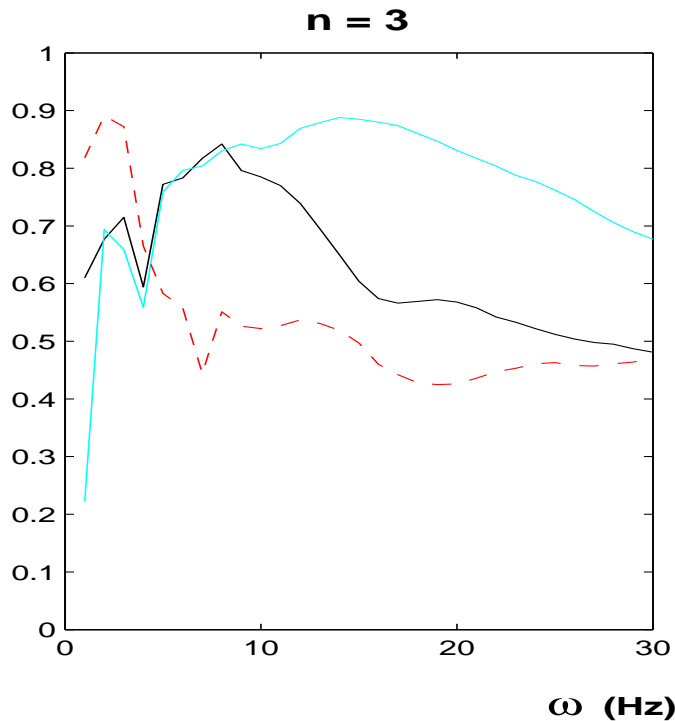

$\gamma$

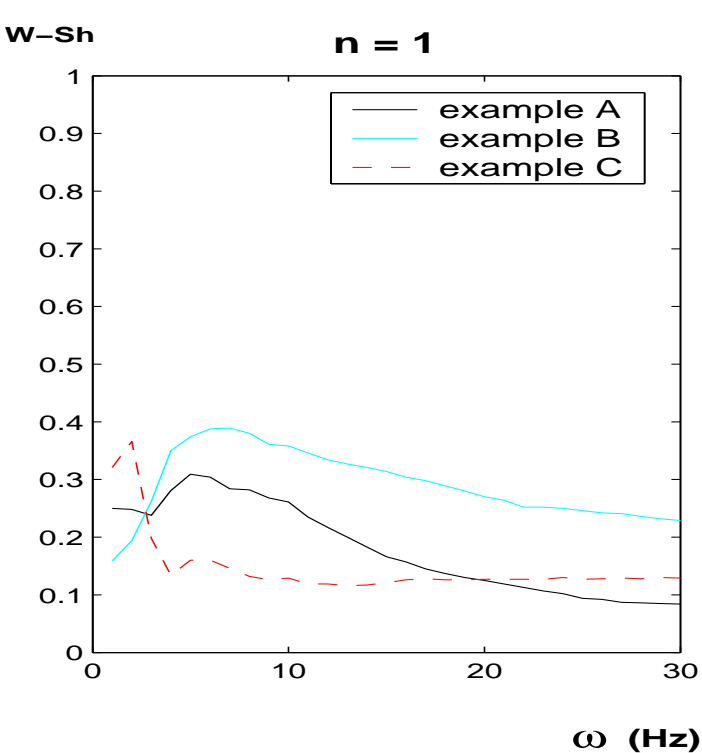

$\gamma$
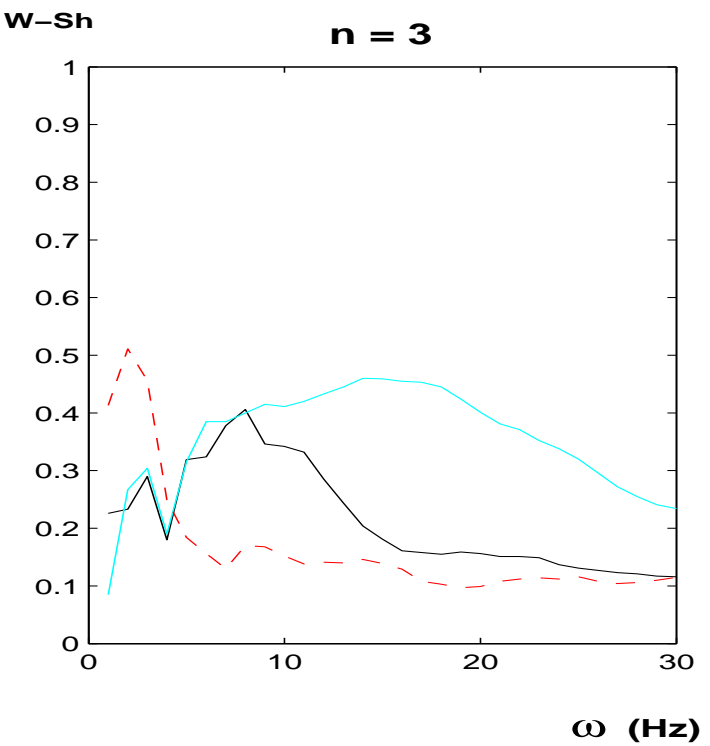

Figure 7: Phase synchronization indices $\gamma_{\mathrm{W}}$ and $\gamma_{\mathrm{W}-\mathrm{Sh}}$ defined from the Wavelet Transform for two different wavelet functions ( $n=1$ and $n=3$ ). 

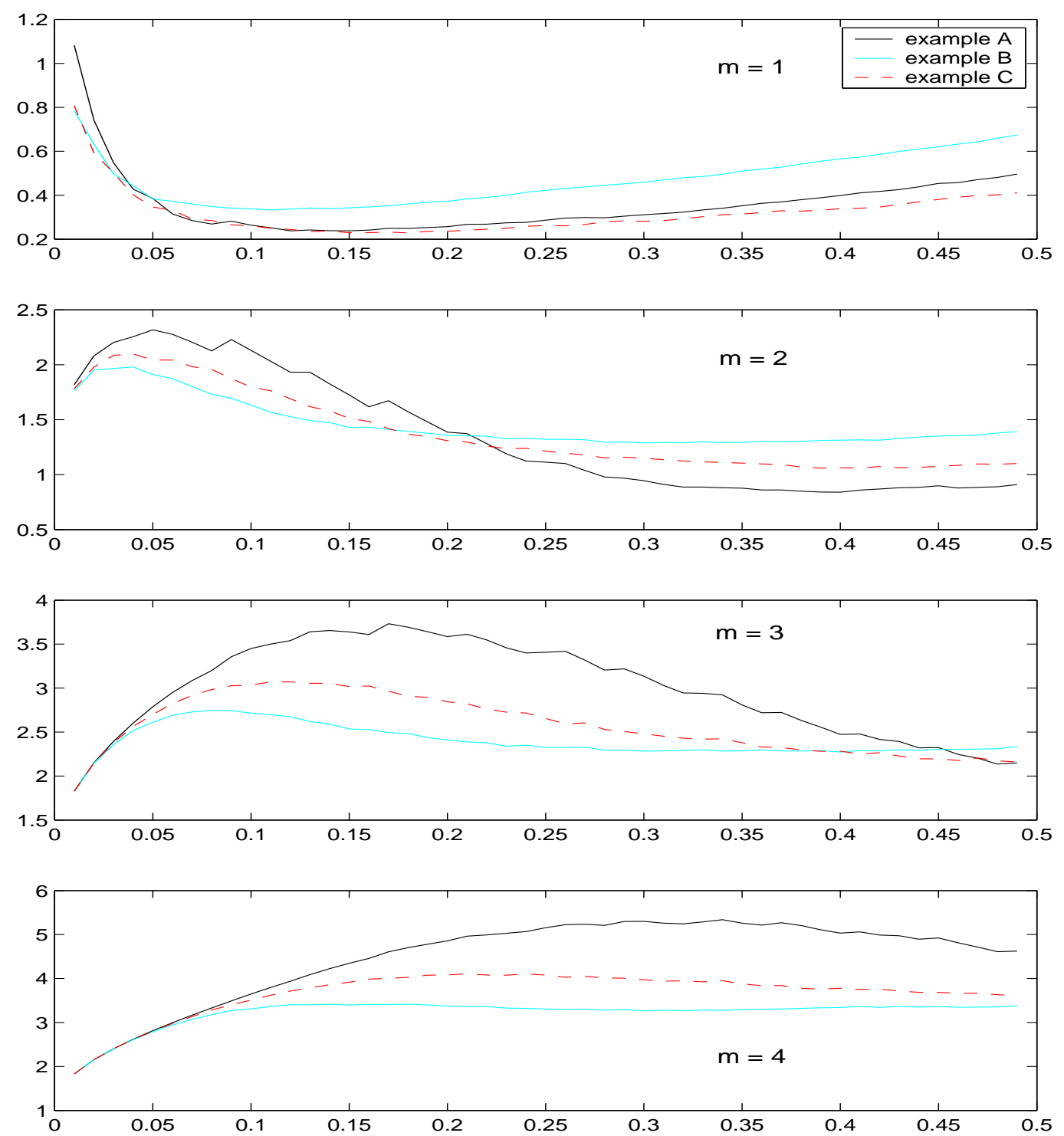

$\delta$

Figure 8: Mutual information calculated with embedding dimensions $m=$ $1,2,3,4$ and varying radius $(\delta)$ of the correlation sum. 